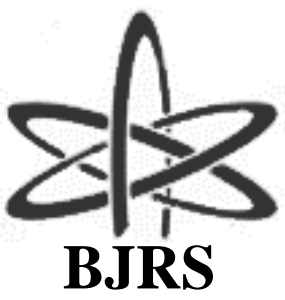
BRAZILIAN JOURNAL
$\mathrm{OF}$
RADIATION SCIENCES

05-03-A (2017) 01-09

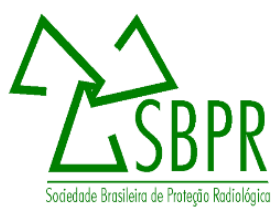

\title{
Comparação entre duas metodologias para correção da uniformidade de fontes extensas de referência
}

\author{
I. A. Silva Junior ${ }^{\mathrm{a}, \mathrm{b}}$; P. T. D. Siqueira ${ }^{\mathrm{b}}$; E. Nascimento ${ }^{\text {c }}$, V. Vivolo ${ }^{\mathrm{b}}$; M. P. A. Potiens ${ }^{\mathrm{b}}$

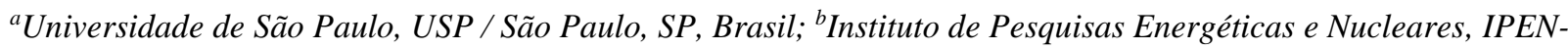

CNEN/SP/São Paulo, SP, Brasil; ' Universidade Federal da Bahia, UFBA / Salvador, BA, Brasil

iremarjr@gmail.com

\section{RESUMO}

São apresentados neste artigo os procedimentos para obtenção de fatores de correção de uniformidade de fontes extensas de referência sugerida por duas metodologias distintas. A primeira metodologia é apresentada pelo Guia de Boas Práticas do $N^{\circ} 14$ do NPL, que oferece uma correção numérica e a segunda é uma correção que utiliza o código de transporte de radiação, MCNP5. Ambos os métodos resultam na proposição de valores de correções muito similares percentualmente, com desvio máximo de $0,24 \%$.

Palavras-chave: Fonte extensa de referência, calibração, correção, uniformidade, MCNP5.

\begin{abstract}
This article presents the procedures to obtain the uniformity correction factors for large-area reference sources proposed by two different methodologies. The first methodology is presented by the Good Practice Guide of $\mathrm{N}^{\mathrm{o}} 14$ of the NPL, which provides a numerical correction. The second one uses the radiation transport code, MCNP5, to obtain the correction factor. Both methods retrieve very similar corrections factor values, with a maximum deviation of $0.24 \%$.
\end{abstract}

Keywords: large-area reference source, calibration, uniformity, correction, MCNP5. 


\section{INTRODUÇÃO}

Fontes extensas de referência são utilizadas em calibrações de monitores de contaminação de superfície. A norma ISO 8769:2016 [1] exige que esse tipo de fonte tenha uma uniformidade superior a $90 \%$.

Há na literatura trabalhos [2,3] que relatam um desacordo entre os valores de uniformidade de fontes extensas de referência usadas pelo IPEN-LCI e as exigências da norma. O Guia de Boas Práticas $\mathrm{N}^{\circ} 14$ do NPL [4] fornece um método numérico para corrigir esse problema encontrado em algumas fontes extensas de referência. Uma proposta alternativa utilizando a simulação do sistema de calibração (fonte-detector) com o código de transporte de radiação ionizante MCNP5 [5] foi desenvolvida com o objetivo de fornecer um fator de correção para essas fontes [6]. Neste trabalho apresenta-se uma comparação entre estes dois métodos: o numérico, dado pelo guia de boas práticas, e o desenvolvido empregando-se a simulação com o código MCNP5.

\section{MATERIAIS E MÉTODOS}

\subsection{Equipamentos}

Neste trabalho foram utilizadas as fontes extensas de referência de ${ }^{14} \mathrm{C},{ }^{99} \mathrm{Tc},{ }^{36} \mathrm{Cl},{ }^{90} \mathrm{Sr}$, com dimensões $10 \times 15 \mathrm{~cm}^{2}$ cada e com datas de referência entre 1996 e 1997 . Nas medidas realizadas foram usados um monitor de radiação da Thermo modelo FH40GX e uma sonda do tipo pancake modelo FHZ732GM.

Para o mapeamento da uniformidade das fontes extensas de referência foi adotado o procedimento de dividir as fontes em 24 regiões independentes, de $2,5 \times 2,5 \mathrm{~cm}^{2}$ cada, distribuídas em 4 linha e 6 colunas. Para medir a contribuição de cada uma dessas regiões foi usada uma máscara de alumínio com espessura de $0,2 \mathrm{~cm}$, com um orifício central com as mesmas dimensões que cada região, isto é, $2,5 \times 2,5 \mathrm{~cm}^{2}$, conforme pode ser visto na figura 1. Esta máscara foi sobreposta à fonte, permitindo a passagem da radiação de cada região individualmente, blindando o detector das emissões das outras 23 regiões. Para auxiliar o posicionamento desta máscara nas medidas de uniformidade, foram confeccionados 24 gabaritos em papel conforme mostrado na figura 2 . 
Figura 1: Máscara em alumínio com abertura no centro.

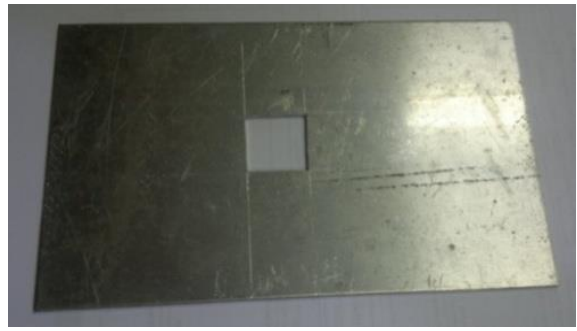

Figura 2: Gabarito em papel com abertura no local para medição.

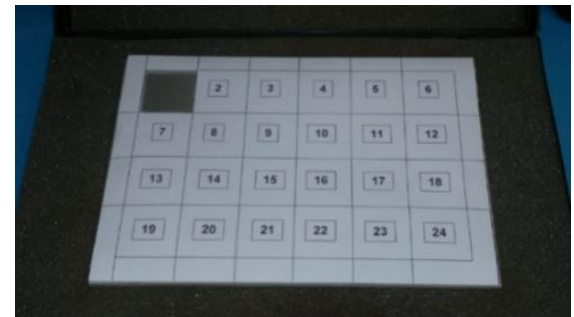

O arranjo experimental usado para as medidas de uniformidade das fontes extensas de referência consistiu de uma base onde se posicionou a fonte extensa de referência, sobreposta a esta um gabarito em papel delimitando uma das 24 regiões de interesse, a máscara de alumínio com o orifício sobreposto ao gabarito, selecionando a região da fonte a ser medida e blindando as outras regiões. A sonda tipo pancake foi posicionada sobre a abertura existente na máscara de forma a permitir a medição apenas da região desejada da fonte. Por questões de proteção radiológica, uma placa de acrílico foi mantida sobre o arranjo durante todo o procedimento de montagem e medida, conforme mostrado na figura 3.

Figura 3: Arranjo experimental.

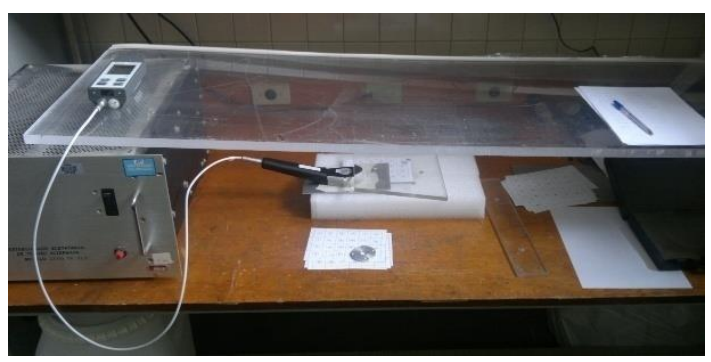




\subsection{Método numérico}

Seguindo a orientação de procedimento apresentado no Apêndice 5 do Guia de Boas Práticas $\mathrm{N}^{\circ} 14$ do NPL [4], foram obtidos os valores de taxa de contagens (cps) para cada uma das 24 regiões definidas anteriormente. Na tabela 1 são apresentados os valores das taxas de contagem das 24 regiões em que foi dividida a fonte extensa de referência de ${ }^{14} \mathrm{C}$, obtidos na avaliação de sua uniformidade.

Tabela 1: Distribuição das taxas de contagem da fonte extensa de referência de ${ }^{14} \mathrm{C}$.

\begin{tabular}{l|l|l|l|l|l}
\hline 2,65 & 1,36 & 1,88 & 1,54 & 2,14 & 5,71 \\
\hline 3,61 & 1,44 & 2,19 & 2,96 & 4,36 & 7,84 \\
\hline 3,29 & 2,55 & 3,55 & 2,50 & 3,49 & 7,45 \\
\hline 3,15 & 2,90 & 3,25 & 3,06 & 3,18 & 3,10 \\
\hline
\end{tabular}

A tabela 2 apresenta os valores das taxas de contagem, apresentados na tabela 1, normalizados pela média das taxas de contagem $\left(\mathrm{E}_{\mathrm{N}}\right)$.

Tabela 2: Distribuição dos valores normalizados das taxas de contagem da fonte de ${ }^{14} \mathrm{C}$.

\begin{tabular}{l|l|l|l|l|l}
\hline 0,80 & 0,41 & 0,57 & 0,47 & 0,65 & 1,73 \\
\hline 1,09 & 0,44 & 0,66 & 0,90 & 1,32 & 2,38 \\
\hline 1,00 & 0,77 & 1,08 & 0,76 & 1,06 & 2,26 \\
\hline 0,96 & 0,88 & 0,99 & 0,93 & 0,96 & 0,94 \\
\hline
\end{tabular}

Embora o Guia de Boas Práticas No 14 do NPL recomende no apêndice 5, seção A5.2 o uso da parte mais uniforme da fonte extensa de referência, quando se tem o mapeamento da uniformidade, optou-se, neste trabalho, pela utilização da parte central das fontes, pois essa é a região comumente usada no processo de calibração. A sonda tipo pancake tem uma área sensível de $15,5 \mathrm{~cm}^{2}$, abrangendo parcialmente as quatro regiões centrais, que formam um quadrado de $5,0 \times 5,0 \mathrm{~cm}^{2}$. A fonte extensa de referência de ${ }^{14} \mathrm{C}$ utilizada está em desacordo com as exigências da norma ISO 8769:2016, pois apresenta um valor de uniformidade de 50,3\%. A figura 4 apresenta um esquema com as 24 regiões na qual foi segmentada a fonte extensa de referência e a indicação da área sensível do detector sobreposta às quatro regiões centrais. 
Figura 4: Desenho esquemático da sobreposição da área sensível do detector à fonte extensa de referência segmentada em 24 regiões.

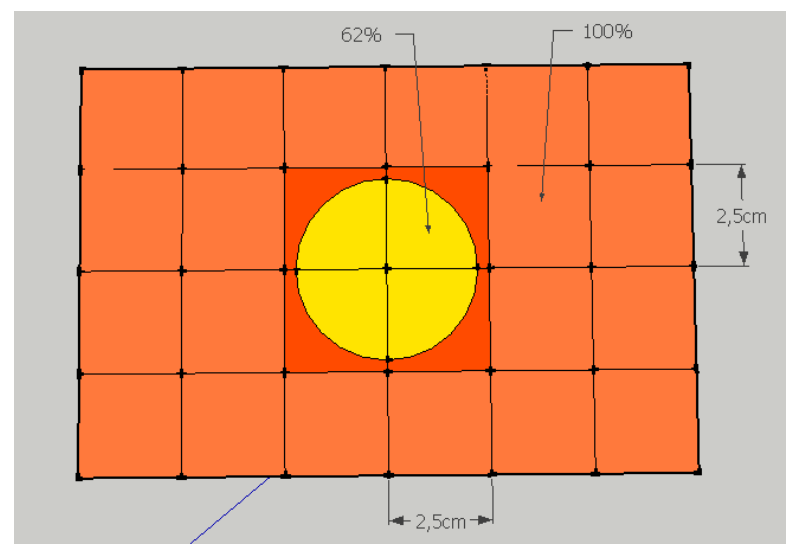

Conforme indicado na figura 4 , a região sensível do detector sobrepõe-se a $62 \%$ da área ativa de cada uma das quatro porções centrais.

São apresentados a seguir os cálculos para obtenção do fluxo efetivo para a região abaixo da área sensível da sonda tipo pancake, conforme procedimentos apresentados no Guia de Boas Práticas $\mathrm{N}^{\circ} 14$ do NPL. Para simplificação na consulta ao Guia, adotou-se neste trabalho a mesma nomenclatura.

Utilizando-se a equação 1, obtém-se o valor do fluxo efetivo $\left(S E R_{\text {eff }}\right)$.

$$
S E R_{e f f}=\frac{\sum_{i}\left(E_{N i} \times f_{i}\right)}{\sum_{i} f_{i}} \times S E R
$$

Onde :

SER: é o valor do fluxo da fonte por unidade de área da fonte toda calculada a partir dos dados do certificado para a região sob a sonda, $E_{N i}$ : é a leitura líquida do instrumento para cada célula sob a sonda e normalizada pela média das leituras líquidas do instrumento de toda a fonte mapeada e $f i$ é a fração de cada célula sob a sonda.

Apresenta-se a seguir como forma ilustrativa, a aplicação da metodologia de cálculo para os dados referentes à fonte extensa de referência de ${ }^{14} \mathrm{C}$.

Utilizando-se a equação 1 para calcular o fluxo efetivo da fonte extensa de referência de ${ }^{14} \mathrm{C}$ temse:

$$
S E R_{e f f\left({ }^{14} C\right)}=\frac{(0,66+0,90+1,08+0,76) \times 0,62}{(0,62+0,62+0,62+0,62)} \times S E R_{\left({ }^{14} C\right)}
$$




$$
S E R_{e f f\left({ }^{14} C\right)}=0,849 \times S E R_{\left({ }^{14} C\right)}
$$

O valor do fluxo para a data das medidas:

$$
\begin{gathered}
S E R_{\left({ }^{14} C\right)}=41,865 \beta \cdot s^{-1} \\
S E R_{e f f\left({ }^{14} C\right)}=0,849 \times 41,865 \\
S E R_{e f f\left({ }^{14} C\right)}=35,544 \beta \cdot s^{-1}
\end{gathered}
$$

A equação 2 é utilizada para calcular a eficiência do instrumento em porcentagem $\left(E_{f}\right)$ :

$$
E_{f}=\frac{(R-B)}{S E R_{e f f}} \times 100
$$

Onde $(R-B)$ é o valor obtido a partir da subtração das contagens de fundo das do valor medido. Para o caso do ${ }^{14} \mathrm{C}$, temos:

$$
\begin{gathered}
E_{f\left({ }^{14} C\right)}=\frac{(9,81-1,35)}{35,544} \times 100 \\
E_{f(14 C)}=23,8 \%
\end{gathered}
$$

$\mathrm{Na}$ tabela 3 são apresentados os valores calculados para todas as fontes extensas de referência avaliadas neste trabalho.

Tabela 3: Valores de eficiência do instrumento calculados para as fontes avaliadas

\begin{tabular}{cccccc}
\hline Radionuclídeos & SER & SER $_{\text {eff }}$ & R-B (cps) & $E_{f}$ & $E_{f N P L}$ \\
\hline${ }^{14} \mathrm{C}$ & 41,865 & 35,544 & 8,46 & $20,2 \%$ & $23,8 \%$ \\
\hline${ }^{99} \mathrm{Tc}$ & 59,103 & 60,230 & 22,43 & $38,0 \%$ & $37,2 \%$ \\
\hline${ }^{36} \mathrm{Cl}$ & 66,441 & 61,928 & 32,35 & $48,7 \%$ & $52,2 \%$ \\
\hline${ }^{90} \mathrm{Sr} /{ }^{90} \mathrm{Y}$ & 87,645 & 89,017 & 49,53 & $56,5 \%$ & $55,6 \%$ \\
\hline
\end{tabular}

Ef: Eficiência do instrumento sem a correção. 
$\mathrm{E}_{\mathrm{fNPL}}$ : Eficiência do instrumento com a correção numérica proposta pelo NPL.

\subsection{Método com MCNP5}

O MCNP5 [5] é um código de transporte de radiação baseado no método de Monte Carlo. Este código permite a simulação dos principais componentes do sistema de calibração, fonte extensa de referência e detector, reproduzindo-se as condições em que a calibração é realizada, i.e., obtendo-se como resultado a estimativa de taxa de contagens no detector. Neste trabalho o MCNP5 é utilizado para calcular a diferença de valores esperados na taxa de contagens do detector para duas condições de fonte, distintas:

- Uniforme, em que a fonte apresenta um valor de uniformidade de $100 \%$ e

- Não uniforme, em que a especificação da fonte no código é dada pelo mapeamento de sua intensidade.

A simulação destas duas condições resulta em estimativas distintas de reposta do sistema, permitindo avaliar e quantificar a interferência da não uniformidade da fonte no procedimento de calibração.

Obtém-se o fator para a correção da uniformidade da fonte extensa de referência a partir da diferença entre o valor obtido pelas simulações para a fonte extensa de referência uniforme e não uniforme, proporcionando uma estimativa mais acurada para a eficiência do detector.

A Tabela 4 apresenta os valores obtidos para a taxa de contagem por simulações para as 2 representações da fonte extensa de referência: uniforme e não uniforme, cujas incertezas relativas são de $0,15 \%$. Apresentam-se também as diferenças percentuais entre as estimativas de eficiência atribuídas e seus respectivos fatores de correção com suas incertezas.

Tabela 4: Valores obtidos com as simulações

\begin{tabular}{|c|c|c|c|c|}
\hline \multirow{3}{*}{ Radionuclídeos } & \multicolumn{2}{|c|}{ Fonte de referência } & \multirow{2}{*}{ Diferença } & \multirow{2}{*}{ Fator de correção } \\
\hline & Uniforme & Não uniforme & & \\
\hline & $\mathrm{cps}$ & $\mathrm{cps}$ & $(\%)$ & \\
\hline${ }^{14} \mathrm{C}$ & 9,61 & 8,15 & $-17,9$ & $1,179 \pm 0,003$ \\
\hline${ }^{99} \mathrm{Tc}$ & 22,73 & 23,19 & 2,0 & $0,980 \pm 0,002$ \\
\hline${ }^{36} \mathrm{Cl}$ & 34,45 & 32,07 & $-7,4$ & $1,074 \pm 0,002$ \\
\hline${ }^{90} \mathrm{Sr} /{ }^{90} \mathrm{Y}$ & 49,79 & 50,44 & 1,3 & $0,987 \pm 0,002$ \\
\hline
\end{tabular}

Na Tabela 5 são apresentados os valores de eficiências para os diversos radionuclídeos, obtidas a partir das medidas realizadas com a sonda tipo pancake, para três casos: cálculo sem correções 
$\left(\mathrm{E}_{\mathrm{f}}\right)$; com correções numéricas propostas pelo NPL no Guia de Boas Práticas No $14\left(\mathrm{E}_{\mathrm{fNPL}}\right)$; com correções numéricas obtidas com o código MCNP5 ( $\left.\mathrm{E}_{\mathrm{fMCNP}}\right)$. São apresentadas as incertezas de um desvio-padrão nos valores da eficiência do instrumento sem a aplicação de correção e na corrigida pelo MCNP5. Os valores de eficiência corrigidos pelo NPL são apresentados sem a avaliação de suas incertezas, pois este cálculo não faz parte do Guia de Boas Práticas $N^{\circ} 14$ de NPL

Tabela 5: Valores de eficiência do instrumento $\left(\mathrm{E}_{\mathrm{f}}\right)$, eficiência do instrumento com correção do NPL ( $\left.\mathrm{E}_{\mathrm{fNPL}}\right)$, eficiência do instrumento corrigidas pelo MCNP5 ( $\left.\mathrm{E}_{\mathrm{fMCNP}}\right)$ e suas incertezas.

\begin{tabular}{ccccc}
\hline Radionuclídeo & $\begin{array}{c}\text { Energia } \\
\text { Máxima } \\
\text { keV }\end{array}$ & $\begin{array}{c}\mathrm{E}_{\mathrm{f}} \\
(\%)\end{array}$ & $\begin{array}{c}\mathrm{E}_{\mathrm{fNPL}} \\
(\%)\end{array}$ & $\begin{array}{c}\mathrm{E}_{\mathrm{fMCNP}} \\
(\%)\end{array}$ \\
\hline${ }^{14} \mathrm{C}$ & 156,5 & $20,2 \pm 0,5$ & 23,8 & $23,8 \pm 0,6$ \\
\hline${ }^{99} \mathrm{Tc}$ & 293,8 & $38,0 \pm 0,8$ & 37,2 & $37,2 \pm 0,7$ \\
\hline${ }^{36} \mathrm{Cl}$ & 709,5 & $48,7 \pm 0,9$ & 52,2 & $52,3 \pm 1,0$ \\
\hline${ }^{90} \mathrm{Sr} /{ }^{90} \mathrm{Y}$ & $545,9 / 2279,8$ & $56,5 \pm 1,0$ & 55,6 & $55,8 \pm 1,0$ \\
\hline
\end{tabular}

Observa-se, a partir dos dados apresentados na tabela 5, que os valores de eficiência obtidos pelas três metodologias ( $E_{f}$ - sem correção; $E_{f N P L}$ - com correção numérica de uniformidade e $E_{f M C N P}$ - com correção de uniformidade via simulação) apresentam uma concordância qualitativa, i.e., a estimativa de eficiência do detector aumenta com o aumento da energia máxima dos $\beta$ das fontes. Observa-se que os valores de eficiência estimados pelas duas metodologias de correção apresentadas neste trabalho são muito próximos entre si, mostrando uma equivalência nas correções propostas. Contudo ao se comparar aos valores de eficiência, sem correção e com correção, chega-se à proposição de valores estatisticamente distintos e eventualmente incompatíveis (casos do ${ }^{14} \mathrm{C}$ e ${ }^{36} \mathrm{Cl}$ ).

\section{CONCLUSÕES}

As avaliações de eficiência de detectores em procedimentos de calibração podem ser significativamente afetadas pela falta de uniformidade da fonte extensa de referência usada no procedimento. Neste trabalho obtiveram-se valores de eficiência que diferiram em até 15\% (3 em 20) para a fonte de ${ }^{14} \mathrm{C}$, que é, entre as fontes estudadas, aquela com menor valor de uniformidade o que 
evidencia a importância da adoção de procedimentos de correção para fontes extensas de referência sem a uniformidade recomendada pela ISO8769:2016. As metodologias de correção adotadas neste trabalho apresentaram estimativas de eficiência praticamente iguais para todos os valores, e as poucas que desviam um pouco, estão dentro de um desvio-padrão, demonstrando concordância entre ambas as estimativas de correção da eficiência..

\section{AGRADECIMENTOS}

Os autores agradecem ao Conselho Nacional de Desenvolvimento Científico e Tecnológico (CNPq), à Comissão Nacional de Energia Nuclear (CNEN) e à Coordenação de Aperfeiçoamento de Pessoal de Nível Superior (CAPES) pelo apoio financeiro parcial.

\section{REFERÊNCIAS}

1. ABNT NBR ISO 8769 - Associação Brasileira de Normas Técnicas. Fontes de referência - Calibração de monitores de contaminação de superfície - Emissores de alfa, beta e fótons. Rio de Janeiro - RJ: 2016.

2. VIVOLO V. and POTIENS, M. P. A. Evaluation of the planar sources surface homogeneity used to instruments calibration. Applied Radiation and Isotopes 68, 605606. 2010.

3. Silva JÚNIOR, I. A., XAVIER, M., SIQUEIRA, P. T. D. e POTIENS, M. P. A. Avaliação da homogeneidade das fontes planas de referência utilizadas na calibração de monitores de contaminação de superfície.International Joint Conference- RADIO 2014.

4. LEE, C. J. and BURGESS, P. H. The examination, testing and calibration of portable radiation protection instruments. Measurement Good Practice Guide No14. Issue 2. NPL (National Physical Laboratory), 2014.

5. X-5 Monte Carlo Team, MCNP - Version 5, Vol. I: Overview and Theory, MC Team, LA-UR-03-1987 (2003).

6. SILVA JÚNIOR, I. A., SIQUEIRA, P. T. D., XAVIER, M., NASCIMENTO E. e POTIENS, M. P. A. Overcoming the reference large-area sources non-uniformity in surface area monitor calibration. International Nuclear Atlantic Conference - INAC 2015. 